\title{
Augmented reality navigation for spinal pedicle screw instrumentation using intraoperative $3 \mathrm{D}$ imaging
}

\author{
Müller, Fabio ; Roner, Simon ; Liebmann, Florentin ; Spirig, José M ; Fürnstahl, Philipp ; Farshad,
} Mazda

\begin{abstract}
BACKGROUND CONTEXT Due to recent developments in augmented reality with headmounted devices, holograms of a surgical plan can be displayed directly in the surgeon's field of view. To the best of our knowledge, three dimensional (3D) intraoperative fluoroscopy has not been explored for the use with holographic navigation by head-mounted devices in spine surgery. PURPOSE To evaluate the surgical accuracy of holographic pedicle screw navigation by head-mounted device using 3D intraoperative fluoroscopy. STUDY DESIGN In this experimental cadaver study, the accuracy of surgical navigation using a head-mounted device was compared with navigation with a state-of-the-art posetracking system. METHODS Three lumbar cadaver spines were embedded in nontransparent agar gel, leaving only commonly visible anatomy in sight. Intraoperative registration of preoperative planning was achieved by 3D fluoroscopy and fiducial markers attached to lumbar vertebrae. Trackable custom-made drill sleeve guides enabled real-time navigation. In total, $20 \mathrm{~K}$-wires were navigated into lumbar pedicles using AR-navigation, $10 \mathrm{~K}$-wires by the state-of-the-art pose-tracking system. 3D models obtained from postexperimental CT scans were used to measure surgical accuracy. MF is the founder and shareholder of Incremed AG, a Balgrist University Hospital start-up focusing on the development of innovative techniques for surgical executions. The other authors declare no conflict of interest concerning the contents of this study. No external funding was received for this study. RESULTS No significant difference in accuracy was measured between AR-navigated drillings and the gold standard with pose-tracking system with mean translational errors between entry points ( $3 \mathrm{D}$ vector distance; $\mathrm{p}=.85$ ) of $3.4 \pm 1.6 \mathrm{~mm}$ compared with $3.2 \pm 2.0 \mathrm{~mm}$, and mean angular errors between trajectories ( $3 \mathrm{D}$ angle; $\mathrm{p}=.30)$ of $4.3^{\circ} \pm 2.3^{\circ}$ compared with $3.5^{\circ} \pm 1.4^{\circ}$. CONCLUSIONS In conclusion, holographic navigation by use of a head-mounted device achieve accuracy comparable to the gold standard of high-end pose-tracking systems. CLINICAL SIGNIFICANCE These promising results could result in a new way of surgical navigation with minimal infrastructural requirements but now have to be confirmed in clinical studies.
\end{abstract}

DOI: https://doi.org/10.1016/j.spinee.2019.10.012

Posted at the Zurich Open Repository and Archive, University of Zurich ZORA URL: https://doi.org/10.5167/uzh-177277

Journal Article

Accepted Version

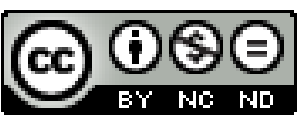

The following work is licensed under a Creative Commons: Attribution-NonCommercial-NoDerivatives 4.0 International (CC BY-NC-ND 4.0) License. 
Originally published at:

Müller, Fabio; Roner, Simon; Liebmann, Florentin; Spirig, José M; Fürnstahl, Philipp; Farshad, Mazda (2020). Augmented reality navigation for spinal pedicle screw instrumentation using intraoperative 3D imaging. The Spine Journal, 20(4):621-628.

DOI: https://doi.org/10.1016/j.spinee.2019.10.012 


\section{Augmented Reality Navigation for Spinal Pedicle Screw Instrumentation using Intraoperative 3D Imaging}

RUNNING TITLE: AR navigation using imaging registration

Fabio Müller ${ }^{\mathrm{a},{ }^{*}} ;$ Simon Roner $^{\mathrm{a}}$; Florentin Liebmann ${ }^{\mathrm{b}, \mathrm{c}}$; José M. Spirig $^{\mathrm{a}}$; Philipp Fürnstahl ${ }^{\mathrm{b}}$; Mazda Farshad ${ }^{\mathrm{a}}$

a Department of Orthopaedics, Balgrist University Hospital, University of Zurich, Forchstrasse 340, 8008 Zurich, Switzerland

${ }^{\mathrm{b}}$ Computer Assisted Research and Development Group, Balgrist University Hospital, University of Zurich, Lengghalde 5, 8008 Zurich, Switzerland

${ }^{c}$ Laboratory for Orthopaedic Biomechanics, ETH Zurich, Forchstrasse 328, 8008 Zurich, Switzerland

* CORRESPONDENCE TO

Fabio Müller

University Hospital Balgrist

Forchstrasse 340

8008 Zurich

Switzerland

Phone: +41 (0)44 38611 11; Fax: +41 (0)44 3861109

E-mail: fabio.mueller3@uzh.ch 


\section{ACKNOWLEDGMENTS}

This work is part of "SURGENT" under the auspices of University Medicine Zurich / Hochschulmedizin Zürich. Imaging was performed with the support of the Swiss Center for Musculoskeletal Imaging, SCMI, Balgrist Campus AG, Zurich. We thank Fabio Carrillo and Jonas Walker for their great support throughout the experiments, especially during the design of custom-made guides and markers as well as illustrations.

\section{ABSTRACT}

\section{Background context:}

Due to recent developments in augmented reality with head-mounted devices, holograms of a surgical plan can be displayed directly in the surgeon's field of view. To the best of our knowledge, 3D intraoperative fluoroscopy has not been explored for the use with holographic navigation by head-mounted devices in spine surgery.

\section{Purpose:}

To evaluate the surgical accuracy of holographic pedicle screw navigation by head-mounted device using 3D intraoperative fluoroscopy.

Study design:

In this experimental cadaver study, the accuracy of surgical navigation using a head-mounted device was compared to navigation with a state-of-the-art pose-tracking system.

\section{Methods:}

Three lumbar cadaver spines were embedded in non-transparent agar gel, leaving only commonly visible anatomy in sight. Intraoperative registration of preoperative planning was 
achieved by 3D fluoroscopy and fiducial markers attached to lumbar vertebrae. Trackable custom-made drill sleeve guides enabled real-time navigation. In total, $20 \mathrm{~K}$-wires were navigated into lumbar pedicles using AR-navigation, $10 \mathrm{~K}$-wires by the state-of-the-art posetracking system. 3D models obtained from post-experimental CT scans were used to measure surgical accuracy. MF is founder and shareholder of Incremed AG, a Balgrist University Hospital start-up focusing on the development of innovative techniques for surgical executions. The other authors declare no conflict of interest concerning the contents of this study. No external funding was received for this study. Results:

No significant difference in accuracy was measured between AR-navigated drillings and the gold standard with pose-tracking system with mean translational errors between entry points (3D vector distance; $\mathrm{p}=0.85$ ) of $3.4 \pm 1.6 \mathrm{~mm}$ compared to $3.2 \pm 2.0 \mathrm{~mm}$, and mean angular errors between trajectories ( $3 \mathrm{D}$ angle; $\mathrm{p}=0.30$ ) of $4.3 \pm 2.3^{\circ}$ compared to $3.5 \pm 1.4^{\circ}$.

\section{Conclusion:}

In conclusion, holographic navigation by use of a head-mounted device achieves an accuracy comparable to the gold standard of high-end pose-tracking systems.

\section{Clinical significance:}

These promising results could result in a new way of surgical navigation with minimal infrastructural requirements but now have to be confirmed in clinical studies.

KEYWORDS: augmented reality; surgical navigation; HoloLens; pedicle screw; 3D surgical accuracy; spine; surgical accuracy. 


\section{INTRODUCTION}

The proximity of vital and neural structures in spine surgery requires very high accuracy from the surgeons [1]. Recently, there has been increasing interest in augmented reality $\left(\mathrm{AR}^{1}\right)$ and its potential to improve accuracy of orthopaedic surgeries [2-5]. To perform surgical navigation by holographic guidance, a three-dimensional $\left(3 \mathrm{D}^{2}\right)$ preoperative plan can be uploaded to the AR head-mounted device $\left(\mathrm{HMD}^{3}\right)$ and registered (superimposed) with the intraoperative anatomy [6]. HMDs enable visualization of a 3D hologram directly into the surgeon's field of view. Compared to current state-of-the-art pose-tracking systems $\left(\mathrm{PTS}^{4}\right)$ working with two-dimensional $\left(2 \mathrm{D}^{5}\right)$ monitors, HMDs have the advantage of direct overlay of navigation on anatomy and that the surgeon can stay focused on the operating field without having to turn away.

A systematic review of most recent AR advancements reported a surgical accuracy between $1 \mathrm{~mm}$ to $5 \mathrm{~mm}$ and attested improvements in safety and efficacy of surgical procedures $[2,6,7]$. In a comparison of three different HMDs, Qian et al. presented the HoloLens (Microsoft, Redmond, USA) to be most suitable for supporting surgical interventions [8]. Since the position of the patient's anatomy in surgery is different from the position in the preoperatively acquired computed tomography $\left(\mathrm{CT}^{6}\right)$, the anatomy can be registered

\footnotetext{
Abbreviations

${ }^{1}$ AR: augmented reality

2 3D: three-dimensional

${ }^{3}$ HMD: head-mounted device

${ }^{4}$ PTS: pose-tracking system

${ }^{5}$ 2D: two-dimensional

${ }^{6} \mathrm{CT}$ : computed tomography

${ }^{7}$ ICP: itierative closest point

${ }^{8}$ DG: drill sleeve guide

${ }^{9}$ TE: translational error

${ }^{10} \mathrm{AE}$ : angular error

${ }^{11}$ SD: standard deviation
} 
intraoperatively in order to align it with the hologram of the 3D preoperative plan [9]. Several registration techniques such as manual or landmark-based surface registration, surface digitalization or intraoperative ultrasound have been employed $[5-7,9,10]$. However, in conventional navigation of spine surgeries, imaging-based registration remains the gold standard to perform the registration task precisely, and 3D fluoroscopy has been shown to be a reliable imaging technique in this regard [11]. Surprisingly, to the best of our knowledge, imaging-based registration has not been investigated for holographic navigation and compared to a state-of-the-art real-time PTS.

In this cadaver study, AR-enabled holographic navigation of pedicle screw instrumentation by use of an HMD (HoloLens, Microsoft, Redmond, USA) was compared to gold standard navigation with a state-of-the-art PTS (fusionTrack500, Atracsys LLC, Puidoux, Switzerland). Intraoperative registration by $3 \mathrm{D}$ fluoroscopy was used in both methods to ensure identical experimental setups and comparable results.

\section{MATERIAL AND METHODS}

This experimental cadaver study was approved by the Cantonal Ethics Committee on research involving humans (ID: KEK 2017-00874). Consent to use a person's body for research purposes was given by the person itself or his/her representative. After the experiments, all exogenic material was extracted and cadavers were cremated.

\section{Study design:}

Three isolated and fresh-frozen lumbar cadaver spines ranging from T12 to coccyx, with intact and healthy anatomy, shortened ribs and autochthonic spinal muscles, were acquired from Science Care (Phoenix, USA). On each spine, all five lumbar vertebrae were planned to 
be instrumented by pedicle screws on both sides, resulting in 10 pedicles per spine. Two groups were formed: The "AR group", representing the study group, comprised two cadaver spines with 20 pedicle screws navigated by AR hologram guidance through an HMD. The control group included one cadaver spine with 10 pedicle screws navigated by a state-of-theart PTS and was called "PTS group". Due to limited availability of specimens, the smaller sample size of the control group was assumed to be justifiable considering also the vast amount of literature reporting reliability and small divergence in the performance of surgical navigation with PTS. All lumbar pedicles were instrumented by an experienced senior surgeon (M. F.). Instead of pedicle screws, K-wires (diameter: $1.5 \mathrm{~mm}$ ) were inserted.

\section{Experimental setup:}

CT scans with a slice thickness of $1 \mathrm{~mm}$ (SOMATOM Edge Plus, Siemens Healthcare GmbH, Erlangen, Germany) were obtained in supine position without fixation of specimens. Semiautomatic segmentation of CT data was conducted by commercial software (Mimics 19.0, Materialise NV, Leuven, Belgium) to generate 3D triangular surface bone models. The anatomical coordinate system of each vertebra was defined by $\mathrm{x}=$ sagittal axis (anteroposterior; red arrow in Figure 1), $y=$ coronal axis (mediolateral, green arrow in

Figure 1), $\mathrm{z}=$ longitudinal axis (craniocaudal; blue arrow in Figure 1). Preoperative planning of trajectories for lumbar pedicle screws from L1 to L5 was executed using the preoperative planning software CASPA (Balgrist CARD AG, Zurich, Switzerland). Trajectories were planned by an experienced spine surgeon (M. F.) according to the traditional Weinstein technique [12]: Screw trajectories were planned in the center of the pedicles, being parallel to the superior endplates of the vertebrae. The screw entry points were determined by calculating the intersection between the central cylindrical screw 
trajectory (diameter: $2 \mathrm{~mm}$ ) and the posterior surface of the vertebra, lateral to the superior articular facet.

The autochthonic spinal muscles were dissected and removed to gain access to the spine mimicking a standard surgical approach from posterior. The lumbar spines were embedded in non-transparent agar gel (Repligel-PG, dent-e-con e.K., Lonsee, Germany) in a prone position. The setup was covered with surgical drapes such that only the vertebral surface visible in an open posterior approach was left uncovered. For the AR group, sterile commercial fiducial registration markers (IMAGE LOCK VisiMARKER, CLEAR GUIDE MEDICAL, Baltimore, USA) were attached to custom-made frames and fixed onto the spinous processes by three K-wires $(1.2 \mathrm{~mm})$. Due to space constraints, not every vertebra in the study group was equipped with markers. The spinous process of L1, L3 and L5 for one cadaver spine and L2 and L4 for the other were mounted with registration markers. Therefore, 5 lumbar vertebrae were registered with a marker attached to their corresponding spinous process, whereas 5 had a registration marker on the neighboring spinous process (Figure 2a). For the PTS group, sterile commercial infrared-reflective spheres (model 2010-03; navigation marker Snap, ILUMARK GmbH, Feldkirchen b. München, Germany; diameter $11 \mathrm{~mm}$ ) were attached to custom-made frames and fixed laterally through the agar gel to each lumbar vertebral body, on the opposite side of the surgeon. Every marker was pinned with two Kwires (Figure 2b).

The registration markers of both groups were equipped with four titanium spheres each (model TI006830; Goodfellow Cambridge Limited, Huntingdon, UK) being visible in 3D fluoroscopy. Intraoperative imaging by 3D fluoroscopy (Ziehm Vision RFD 3D, Ziehm Imaging $\mathrm{GmbH}$, Nuremberg, Germany; slice thickness $1 \mathrm{~mm}$ ) was obtained from each specimen. For registration between CT and 3D fluoroscopy, the titanium spheres and the 
vertebrae were semi-automatically segmented from the 3D fluoroscopy data and imported into the CASPA software. The registration process is illustrated in Figure 1. In a first step, the registration marker's coordinate system was calculated using the titanium spheres (Figure 1a bottom; bright dots). Thereby, the current marker's position (Figure 1b bottom; light green) relative to the intraoperative anatomy was determined. In a second step, iterative closest point $\left(\mathrm{ICP}^{7}\right)$ was applied similar to Liebmann et al. to align the preoperative with the intraoperative anatomy [6], yielding the corresponding coordinate system transformation of the vertebrae. Based on the transformations of the marker relative to the intraoperative vertebra and the transformation between pre- and intra-operative anatomy, the correspondence between preoperative plan and intraoperative situation was established (Figure 1c).

After registration to each cadaver specimen, the 3D preoperative planning of the desired level was displayed, either by superimposing the real anatomy with a hologram (AR group) or by displaying it on a screen facing the surgeon (PTS group). Marker positions were tracked in real-time, compensating motion during instrumentation. To increase processing speed, unused markers were covered (AR group) and reflective spheres were only mounted onto the marker corresponding to the level to be worked on (PTS group). The PTS was placed cranial to the cadaver spine. In both groups, navigation of K-wire insertion was performed with a trackable drill sleeve guide $\left(\mathrm{DG}^{8}\right)$, similar to the approach of Liebmann et al., except that infraredreflective spheres were used for tracking with the PTS (Figure 3a and b) [6].

Navigation was conducted in two steps. First, entry points of the pedicle screws were displayed by blue spheres with diameters of $2 \mathrm{~mm}$ (Figure 4a). Additionally, the Euclidean distance between the planned entry point and current tip position of the DG was calculated and displayed in millimeters next to the DG. Secondly, a triangle illustrating the deviation 
between the planned trajectory (Figure $\mathbf{4 b}$ ) and the trajectory of the DG was displayed. The corresponding 3D angle in degrees indicated the accuracy of the alignment [6]. The surgeon was additionally supported by the colors of the triangle, illustrating the quality of the alignment. Red indicated that the distance or the 3D angle of the current trajectory was deviating more than $2 \mathrm{~mm}$ or $3^{\circ}$, respectively, whereas green represented less than $2 \mathrm{~mm}$ or $3^{\circ}$, respectively. Hand gestures, performing a double-tap with thumb and index finger, were used to switch between insertion point and trajectory navigation in the AR group, whereas navigation in the PTS group was controlled by a second person using the computer keyboard. Each specimen was instrumented from cranial to caudal, always starting on the right pedicle.

After completion of the experiments, the surgeon filled in a questionnaire rating the following evaluation points from 1 (very bad) to 5 (very good): visual impression of registration accuracy, comfort of usability, intuitiveness of system, visualization of entry points, visualization of trajectory navigation, time expenditure, stability of system, feasibility for surgery, preserved sterility of surgical site.

\section{CALCULATION}

$3 D$ evaluation method:

Post-experimentally, CT scans with $1 \mathrm{~mm}$ slice thickness of the three cadaver specimens were conducted in prone position embedded within agar gel. Vertebrae and K-wires were semiautomatically segmented and registered with the 3D planning, as explained before [6]. 
With the CASPA software, cylinders of $2 \mathrm{~mm}$ diameter were aligned with the 3D models of the K-wires and sphere centers (diameter $2 \mathrm{~mm}$ ), representing the entry points, were placed where the cylinder passed through the posterior surface of the vertebral arch (Figure 5a). The $3 \mathrm{D}$ translational error $\left(\mathrm{TE}^{9}\right)$ of the entry point navigation was calculated by the $3 \mathrm{D}$ Euclidean distance between the centers of the spheres representing planned $\left(x_{1}, y_{1}, z_{1}\right)$ and executed $\left(x_{2}, y_{2}, z_{2}\right)$ entry points using the following formula [13]:

$T E=\sqrt{\left(x_{1}-x_{2}\right)^{2}+\left(y_{1}-y_{2}\right)^{2}+\left(z_{1}-z_{2}\right)^{2}}$

For the calculation of the $3 \mathrm{D}$ angular error $\left(\mathrm{AE}^{10}\right)$, the direction vector of the planned $(\vec{A})$ and executed $(\vec{B})$ trajectories were used and the following formula was applied (Figure 5b) [13]:

$A E=\cos ^{-1}\left(\frac{\vec{A} \cdot \vec{B}}{\|\vec{A}\|\|\vec{B}\|}\right)$

To allow comparison of the outcome with historical data from literature, the method proposed by Guha et al. was implemented as well [14]. Overall time required for navigation was measured from the moment of entry point visualization until the surgeon was finished with Kwire insertion. To assess the influence of registration marker positions in the AR group, the accuracies for levels on which registration markers were directly mounted, were compared to levels navigated by registration markers mounted on neighboring spinous processes.

\section{Statistical analysis:}

Statistical analysis was performed in MATLAB version R2016b (MathWorks, Natick, USA). Metric parameters were presented by mean values and corresponding standard deviation $\left(\mathrm{SD}^{11}\right)$, categorical parameters by frequency and percentage. Comparisons of varying, 
normally distributed parameters were done by Welch's t-test with unequal variance due to unequal sample size. A p-value of $<0.05$ was considered statistically significant.

\section{RESULTS}

$20 \mathrm{~K}$-wires were navigated in the AR group, 10 in the PTS group.

The evaluation of the surgical accuracy resulted in a mean 3D TE in the AR and PTS groups of $3.4 \pm 1.6 \mathrm{~mm}$ and $3.2 \pm 2.0 \mathrm{~mm}$, respectively (Figure 6a). Mean $3 \mathrm{D}$ AE was $4.3 \pm 2.3^{\circ}$ in the AR group and $3.5 \pm 1.4^{\circ}$ in the PTS group (Figure 6b). No statistically significant difference between the groups was found for TE $(p=0.85)$ or $\mathrm{AE}(\mathrm{p}=0.30)$.

In $2 \mathrm{D}$, the axial TE was $1.8 \pm 1.3 \mathrm{~mm}$ (AR group) versus $1.6 \pm 1.0 \mathrm{~mm}$ (PTS group) and the sagittal TE was $2.6 \pm 4.7 \mathrm{~mm}$ versus $0.9 \pm 0.8 \mathrm{~mm}$. The axial $\mathrm{AE}$ was $3.4 \pm 2.5^{\circ}$ and $2.4 \pm$ $1.4^{\circ}$ in the AR and PTS group, respectively. The sagittal AE was $2.1 \pm 1.5^{\circ}$ (AR group) and $2.3 \pm 1.4^{\circ}$ (PTS group)

Two major outliers in pedicles navigated by PTS had to be excluded from the analysis (L2 right and L4 right). In case of L2 right, the surgeon slipped off the vertebral arch during Kwire insertion. Whereas for L4 right, the laterally attached registration marker touched the table during instrumentation of the ipsilateral side, resulting in modification of the registration.

The overall time required for navigation was $57.5 \pm 46.9 \mathrm{~s}$ in the AR group and $45 \pm 16.5 \mathrm{~s}$ in the PTS group $(\mathrm{p}=0.30)$. Within the AR group, no statistically significant difference ( $\mathrm{p}$ value for $\mathrm{TE}=0.63$; $\mathrm{p}$-value for $\mathrm{AE}=0.85$ ) between surgical accuracy of levels on which registration markers were directly attached compared to levels with registration markers 
attached to neighboring vertebrae could be noted. According to the questionnaire (Table 1), the surgeon experienced more intuitive handling and subjectively easier trajectory navigation by using AR.

\section{DISCUSSION}

Although being the gold standard in surgical navigation [11], to our knowledge, image-based registration has not been explored for the use with AR-based HMD and compared to state-ofthe-art navigation systems in spine surgery. In the here performed cadaveric pilot study we found surgical accuracy achieved by AR navigation using HMD with image-based registration to be within the range of commercially available navigation systems. Various different clinical grading systems have been applied to evaluate the outcome of pedicle screw placement, making reproducibility and comparisons between studies difficult $[15,16]$. Only few studies assessing the performance of navigation systems in spinal surgery reported on the surgical accuracy of pedicle screw placement at all $[6,14,17-23]$. Results reported in literature were mostly conducted in 2D [14,17-22]. Kleck et al. used a commercial high-precision PTS for the navigation of pedicle screw placement [23]. They achieved a mean $\mathrm{TE}$ of $5.9 \mathrm{~mm}$ and mean $\mathrm{AE}$ of $3.1^{\circ}$ which is more accurate for $\mathrm{AE}$ but less accurate for $\mathrm{TE}$ compared to our results [23].

To compare our results with 2D error metrics reported in literature, we derived the projected measurements using the method proposed by Guha et al. [14]. Mean axial and sagittal translational errors in $2 \mathrm{D}$ were ranging from $1.3-1.8 \mathrm{~mm}$ and $1.2-1.5 \mathrm{~mm}$ in literature [7,14], whereas other studies did not report on TE [17,20-22]. The 2D accuracy reported ranged from $2.2-4.0^{\circ}$ and $2.6-4.0^{\circ}$ for axial and sagittal AE, respectively [7,14,17,20-22]. 
Elmi-Terander et al. described a higher accuracy, with mean TE of $2.2 \mathrm{~mm}$ and mean AE of $0.9^{\circ}$, using a hybrid operating room [18].

Other experimental studies evaluating different registration methods for AR navigation in orthopaedic surgery have achieved a similar accuracy, but none of those were performed on human cadavers [5-7].

Increasing distance between registration makers and the levels to be instrumented can be a potential source of error if vertebrae move independently during instrumentation $[9,11]$. However, no significant difference in the surgical accuracy was noted in our study, regardless of whether the markers were attached directly on the vertebrae or beside them. This is in line with the findings of Boon Tow et al., reporting about accurate registration of two adjacent spinal levels with a single registration marker [24]. Yet, these findings do not imply that the use of a single registration marker might be sufficiently accurate for the surgical navigation of an entire spine, as it is suggested by some commercially available tracking and navigation systems for spine surgery $[9,24,25]$. Since fewer registration markers result in less preparation time in surgery, experiments assessing the accuracy of AR navigation dependent on the number of registration markers should be considered in future studies.

There have also been no significant differences in navigation times between the AR and the PTS groups. The navigation times measured in our study were even below the results reported in literature ranging from 90-200s $[6,7,18]$. However, it has to be stated that intraoperative registration by $3 \mathrm{D}$ fluoroscopy is time-consuming and segmentation was not fully automated within our study. The time needed for registration has not been added to the reported navigation times. 
The comparison of the surgical accuracy between studies should be handled with care because different evaluation methods had been applied. So far, varying error measures of navigated pedicle screw placement in spine surgery have been reported in literature and no gold standard was established. Our study described a 3D evaluation method to report on surgical accuracy [23]. Liebmann et al. had used the same error measures as our study for the technical evaluation of AR-guided navigation of pedicle screw placement with the Microsoft HoloLens [6]. Besides Liebmann et al., Kleck et al. was the only study reporting the accuracy based on 3D error metrics [6,23]. Contrary, Guha et al. proposed an evaluation based on 2D error measures as a standard for future studies [14]. To improve reporting on surgical accuracy, they suggested measuring the translational and angular error in axial and sagittal planes of CT scans relative to a reference line [14]. In our opinion, a 3D evaluation method is more reliable, since no reader-dependent reference lines or planes need to be defined to measure the projected angles and distances in 2D.

This study faced also limitations of AR navigation using HMD. In the 3D preoperative planning, the entry points and corresponding trajectories were placed directly onto the bony surface. However, some spine surgeons remove parts of the cortical bone with a rongeur at the location of entry to gain easier access to the pedicle. The amount of bone removal cannot be planned preoperatively, and entry point navigation was thereby limited. Further drawbacks have been the use of isolated cadaver spines instead of cadaver torsos as well as the small number of specimens. However, this is the first report of its kind with the design of a pilot study evaluating feasibility and accuracy.

Also, technical limitations currently exist and need to be solved before clinical application; First, transfer of imaging data, segmentation of the intraoperative anatomy and registration 
with the preoperative planning was not performed in an automatic fashion as it would be the case in a real surgery. However, automation of these steps was not the focus of our research as it has already been achieved for other commercial tracking and navigation systems using intraoperative imaging registration $[14,17,18,21-23]$. Secondly, the software application for holographic navigation was unstable, because constant access to internal sensor data through the HoloLens research mode was required [26]. Moreover, simultaneous tracking of two or more markers while the HMD was moving in space, sometimes caused jittering of the hologram and could even provoke application breakdowns requiring restart [6].

Due to the technical limitations mentioned, it has to be stated that the AR navigation by HMD is not yet ready for clinical use. However, despite using hardware that has not primarily been designed for medical purposes, the accuracy of the surgical navigation is surprisingly high and very comparable to high-end optical tracking or navigation systems. Furthermore, studies investigating the performance in a real surgical setting are needed to evaluate the influence of factors such as light conditions, blood, noise disturbance, and breathing artefacts. Since the surgeon is forced to flex his neck more than usual to have the surgical site within the camera's field of view, also ergonomic aspects should be considered in the design of future HMDs.

Our vision of AR-enhanced surgery is an HMD interfacing with all relevant OR devices and thereby acting as a command control interface operated by the surgeon (Figure 7a). Not only navigational assistance but also further important information (e.g. heart rate, blood pressure, blood loss, operation time, imaging) could be visualized within the surgeon's field of view (Figure 7b). Accordingly, AR navigation could reduce infrastructural requirements compared to state-of-the-art navigation systems. Depending on the registration method, at 
least an HMD, fiducial registration markers and a specific DG would be required. (Figures 3a and 7a).

\section{CONCLUSION}

In conclusion, navigation for pedicle instrumentation by augmented reality-based headmounted devices achieves comparable results to state-of-the-art high precision navigation systems when using 3D image-based registration.

\section{REFERENCES}

[1] Rampersaud YR, Simon DA, Foley KT. Accuracy requirements for image-guided spinal pedicle screw placement. Spine (Phila Pa 1976) 2001;26:352-9. doi:10.1097/00007632-200102150-00010.

[2] Vávra P, Roman J, Zonça P, Ihnát P, Němec M, Kumar J, et al. Recent Development of Augmented Reality in Surgery: A Review. J Healthc Eng 2017;2017. doi:10.1155/2017/4574172.

[3] Fischer M, Fuerst B, Lee SC, Fotouhi J, Habert S, Weidert S, et al. Preclinical usability study of multiple augmented reality concepts for K-wire placement. Int J Comput Assist Radiol Surg 2016;11:1007-14. doi:10.1007/s11548-016-1363-x.

[4] Belmustakov S, Bailey C, Weiss CR. Augmented and Virtual Reality Navigation for Interventions in the Musculoskeletal System. Curr Radiol Rep 2018;6:33. doi:10.1007/s40134-018-0293-5.

[5] Ma L, Zhao Z, Chen F, Zhang B, Fu L, Liao H. Augmented reality surgical navigation 
with ultrasound-assisted registration for pedicle screw placement: a pilot study. Int $\mathbf{J}$ Comput Assist Radiol Surg 2017;12:2205-15. doi:10.1007/s11548-017-1652-z.

[6] Liebmann F, Roner S, von Atzigen M, Scaramuzza D, Sutter R, Snedeker J, et al. Pedicle screw navigation using surface digitization on the Microsoft HoloLens. Int $\mathbf{J}$ Comput Assist Radiol Surg 2019;14:1157-65. doi:10.1007/s11548-019-01973-7.

[7] Gibby JT, Swenson SA, Cvetko S, Rao R, Javan R. Head-mounted display augmented reality to guide pedicle screw placement utilizing computed tomography. Int J Comput Assist Radiol Surg 2019;14:525-35. doi:10.1007/s11548-018-1814-7.

[8] Qian L, Barthel A, Johnson A, Osgood G, Kazanzides P, Navab N, et al. Comparison of optical see-through head-mounted displays for surgical interventions with objectanchored 2D-display. Int J Comput Assist Radiol Surg 2017;12:901-10. doi:10.1007/s11548-017-1564-y.

[9] Scheufler K-M, Franke J, Eckardt A, Dohmen H. Accuracy of Image-Guided Pedicle Screw Placement Using Intraoperative Computed Tomography- Based Navigation With Automated Referencing. Part II: Thoracolumbar Spine. Neurosurgery 2011;69:1307-16. doi:10.1227/NEU.0b013e31822ba190.

[10] Urakov TM, Wang MY, Levi AD. Workflow Caveats in Augmented Reality-Assisted Pedicle Instrumentation: Cadaver Lab. World Neurosurg 2019;126:e1449-55. doi:10.1016/j.wneu.2019.03.118.

[11] Du JP, Fan Y, Wu QN, Wang DH, Zhang J, Hao DJ. Accuracy of Pedicle Screw Insertion Among 3 Image-Guided Navigation Systems: Systematic Review and MetaAnalysis. World Neurosurg 2018;109:24-30. doi:10.1016/j.wneu.2017.07.154.

[12] Weinstein JN, Spratt KF, Spengler D, Brick C, Reid S. Spinal pedicle fixation: reliability and validity of roentgenogram-based assessment and surgical factors on 
successful screw placement. Spine (Phila Pa 1976) 1988;13:1012-8.

[13] Schneider PJ, Eberly DH. Geometric tools for computer graphics - The Morgan Kaufmann Series in Computer Graphics and Geometric Modeling. San Francisco: Morgan Kaufmann Publishers; 2003.

[14] Guha D, Jakubovic R, Gupta S, Alotaibi NM, Cadotte D, da Costa LB, et al. Spinal intraoperative three-dimensional navigation: correlation between clinical and absolute engineering accuracy. Spine J 2017;17:489-98. doi:10.1016/j.spinee.2016.10.020.

[15] Tian W, Zeng C, An Y, Wang C, Liu Y, Li J. Accuracy and postoperative assessment of pedicle screw placement during scoliosis surgery with computer-assisted navigation: a meta-analysis. Int J Med Robot Comput Assist Surg 2017;13:e1732. doi:10.1002/rcs.1732.

[16] Kosmopoulos V, Schizas C. Pedicle screw placement accuracy: A meta-analysis. Spine (Phila Pa 1976) 2007;32:E111-120. doi:10.1097/01.brs.0000254048.79024.8b.

[17] Miller CA, Ledonio CG, Hunt MA, Siddiq F, Polly DW. Reliability of the Planned Pedicle Screw Trajectory versus the Actual Pedicle Screw Trajectory using Intraoperative 3D CT and Image Guidance. Int J Spine Surg 2016;10:38. doi: $10.14444 / 3038$.

[18] Elmi-Terander A, Nachabe R, Skulason H, Pedersen K, Söderman M, Racadio J, et al. Feasibility and accuracy of thoracolumbar minimally invasive pedicle screw placement with augmented reality navigation technology. Spine (Phila Pa 1976) 2018;43:101823. doi:10.1097/BRS.0000000000002502.

[19] Kotani Y, Abumi K, Ito M, Takahata M, Sudo H, Ohshima S, et al. Accuracy analysis of pedicle screw placement in posterior scoliosis surgery: Comparison between conventional fluoroscopic and computer-assisted technique. Spine (Phila Pa 1976) 
2007;32:1543-50. doi:10.1097/BRS.0b013e318068661e.

[20] Fan Y, Du J, Zhang J, Liu S, Xue X, Huang Y, et al. Comparison of Accuracy of Pedicle Screw Insertion Among 4 Guided Technologies in Spine Surgery. Med Sci Monit 2017;23:5960-8. doi:10.12659/msm.905713.

[21] Oertel MF, Hobart J, Stein M, Schreiber V, Scharbrodt W. Clinical and methodological precision of spinal navigation assisted by 3D intraoperative O-arm radiographic imaging. J Neurosurg Spine 2011;14:532-6. doi:10.3171/2010.10.spine091032.

[22] Mathew JE, Mok K, Goulet B. Pedicle violation and Navigational errors in pedicle screw insertion using the intraoperative O-arm: A preliminary report. Int J Spine Surg 2013;7:e88-94. doi:10.1016/j.ijsp.2013.06.002.

[23] Kleck CJ, Cullilmore I, LaFleur M, Lindley E, Rentschler ME, Burger EL, et al. A new 3-dimensional method for measuring precision in surgical navigation and methods to optimize navigation accuracy. Eur Spine J 2016;25:1764-74. doi:10.1007/s00586-0154235-0.

[24] Boon Tow BP, Yue WM, Srivastava A, Lai JM, Guo CM, Wearn Peng BC, et al. Does Navigation Improve Accuracy of Placement of Pedicle Screws in Single-level Lumbar Degenerative Spondylolisthesis? J Spinal Disord Tech 2015;28:E472-7. doi:10.1097/BSD.0b013e3182a9435e.

[25] Jin M, Liu Z, Qiu Y, Yan H, Han X, Zhu Z. Incidence and risk factors for the misplacement of pedicle screws in scoliosis surgery assisted by $\mathrm{O}$-arm navigationanalysis of a large series of one thousand, one hundred and forty five screws. Int Orthop 2017;41:773-80. doi:10.1007/s00264-016-3353-6.

[26] Microsoft. HoloLens Research Mode 2018. https://docs.microsoft.com/enus/windows/mixed-reality/research-mode (accessed June 13, 2019). 


\section{FIGURE LEGENDS}

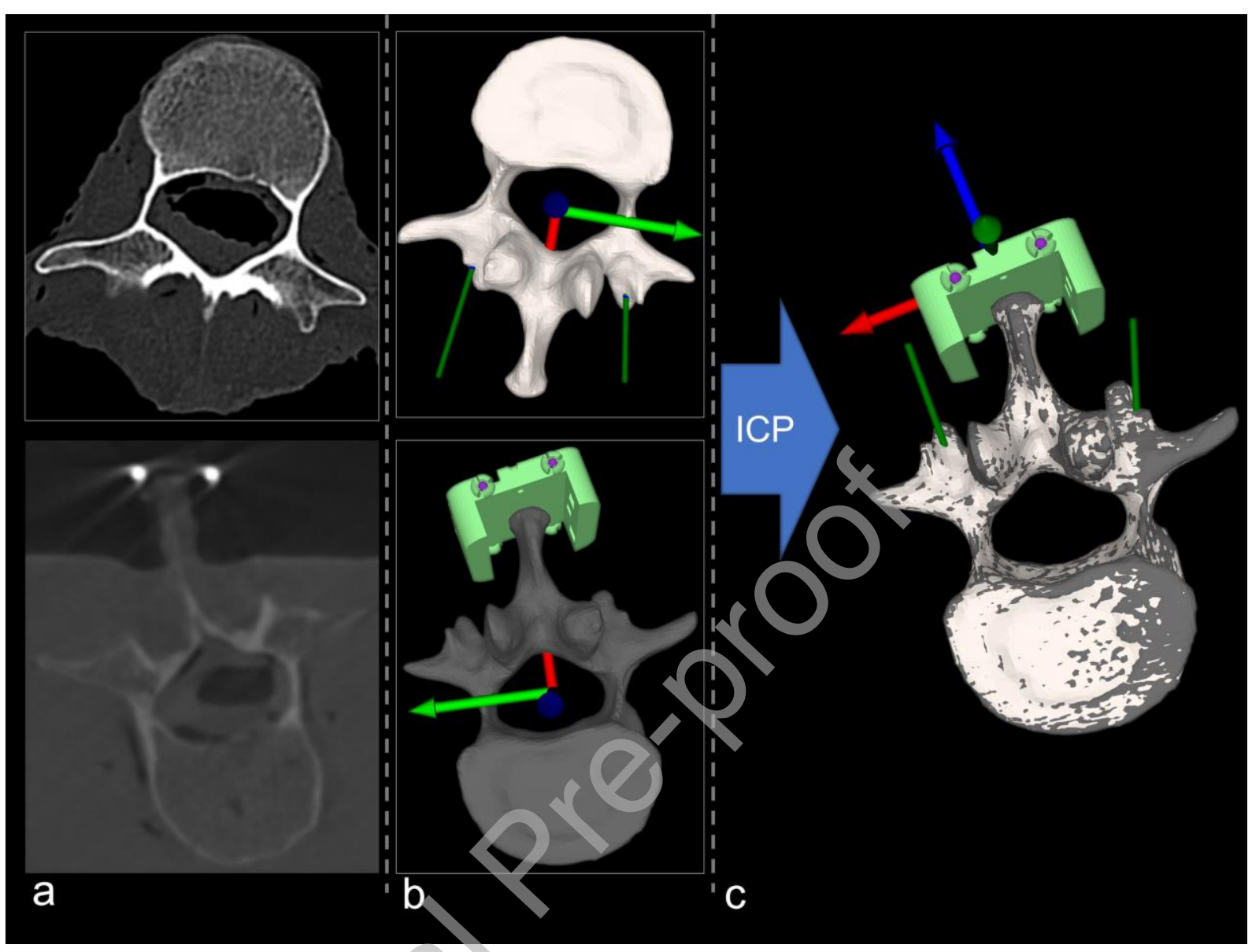

Figure 1-Registration workflow: Surgical planning of entry points (blue spheres) and trajectories (green cylinders) on 3D models (1b top) obtained from preoperative CT scans (1a top). Intraoperative registration using 3D fluoroscopy (1a bottom) to obtain registration marker positions (1b bottom) by titanium spheres. Using iterative closest point (ICP), preoperative planning was semi-automatically aligned with intraoperative anatomy and the corresponding marker's position (1c). 


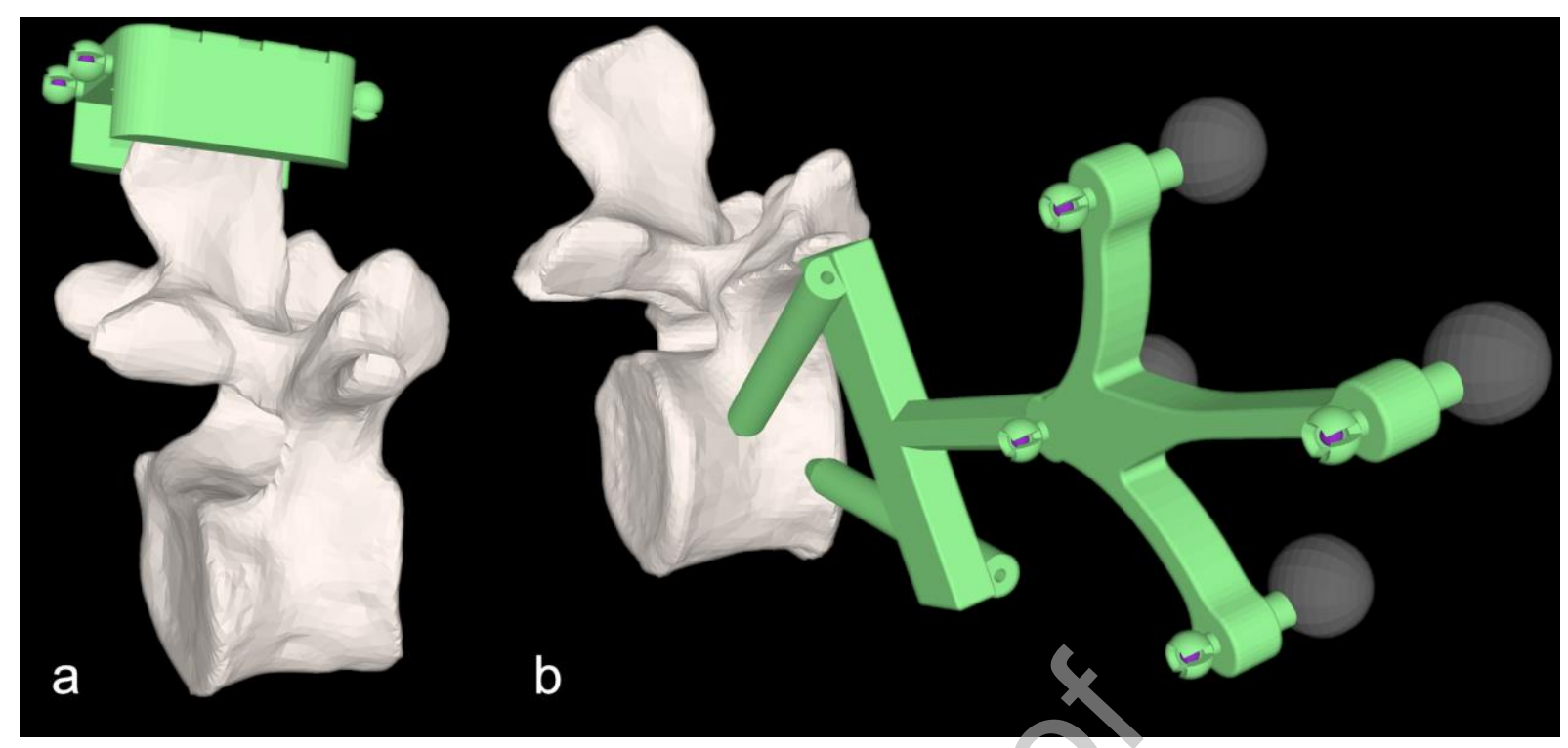

Figure 2 - Registration markers: Experimental setup featuring 3D model of the lumbar spine with custom-made a) fiducial and b) infrared registration markers including titanium spheres (violet spheres).

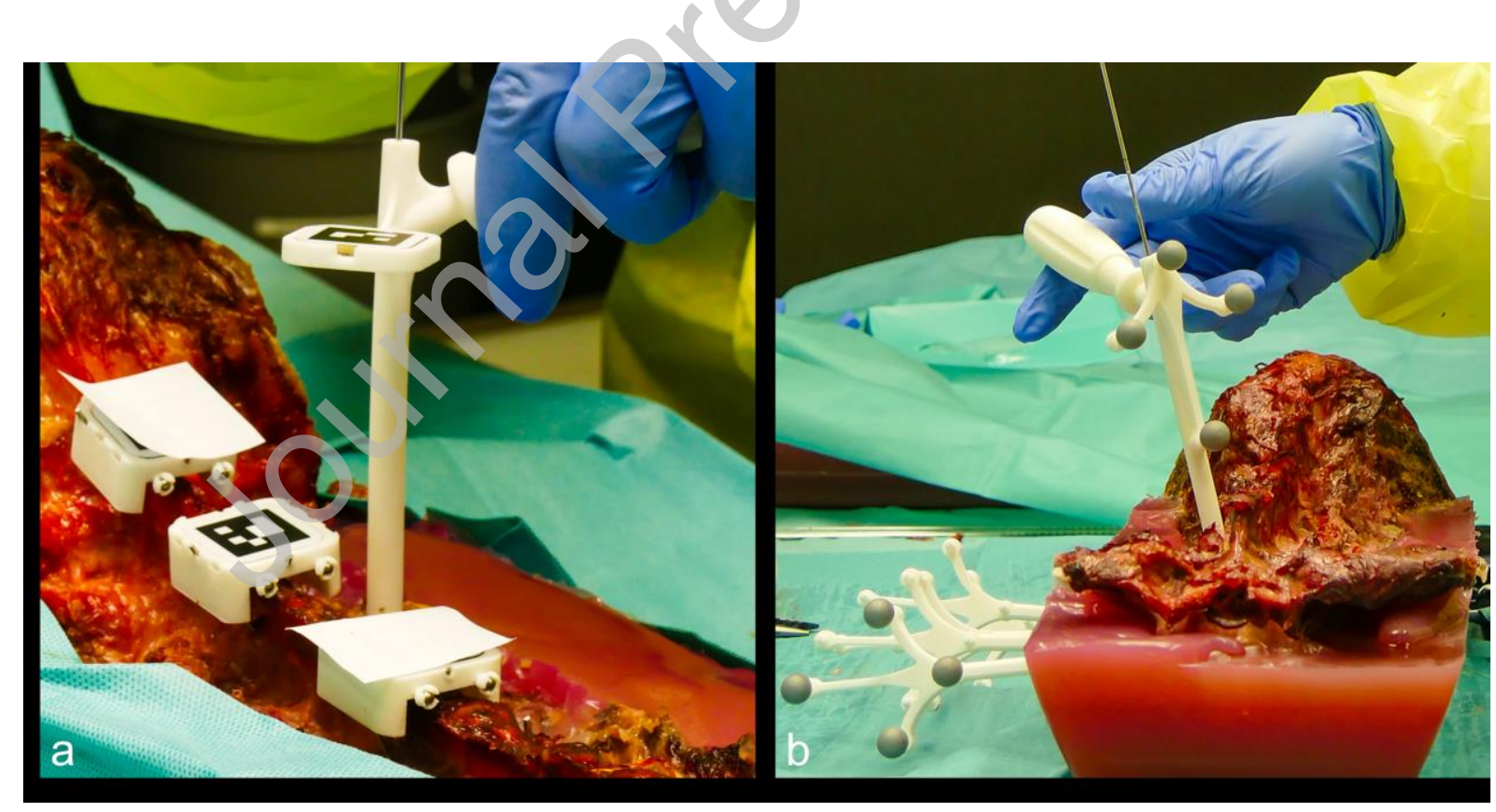

Figure 3 -Drill sleeve guides: Custom-made drill sleeve guides mounted with a) fiducial to navigate K-wire insertion by HMD and b) with reflecting spheres to navigate K-wire insertion by PTS. 


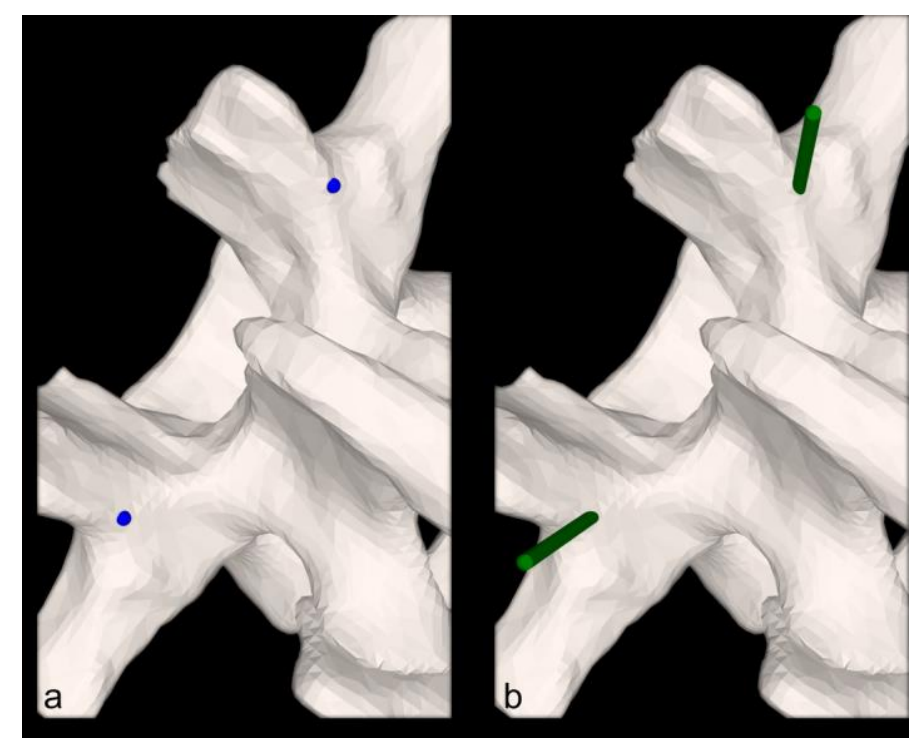

Figure 4 - 3D planning: a) Planned entry points and b) K-wire trajectories on the surface mesh of the vertebral arch.

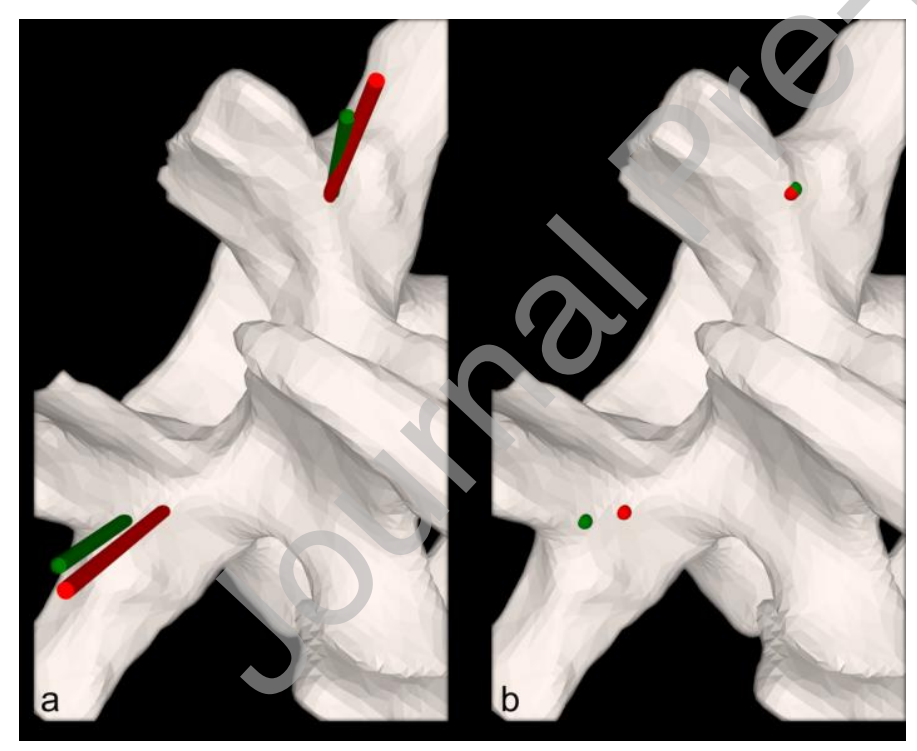

Figure 5 - 3D evaluation method: a) Planned (green) and executed (red) K-wire trajectories and b) entry points on the surface mesh of the vertebral arch. 

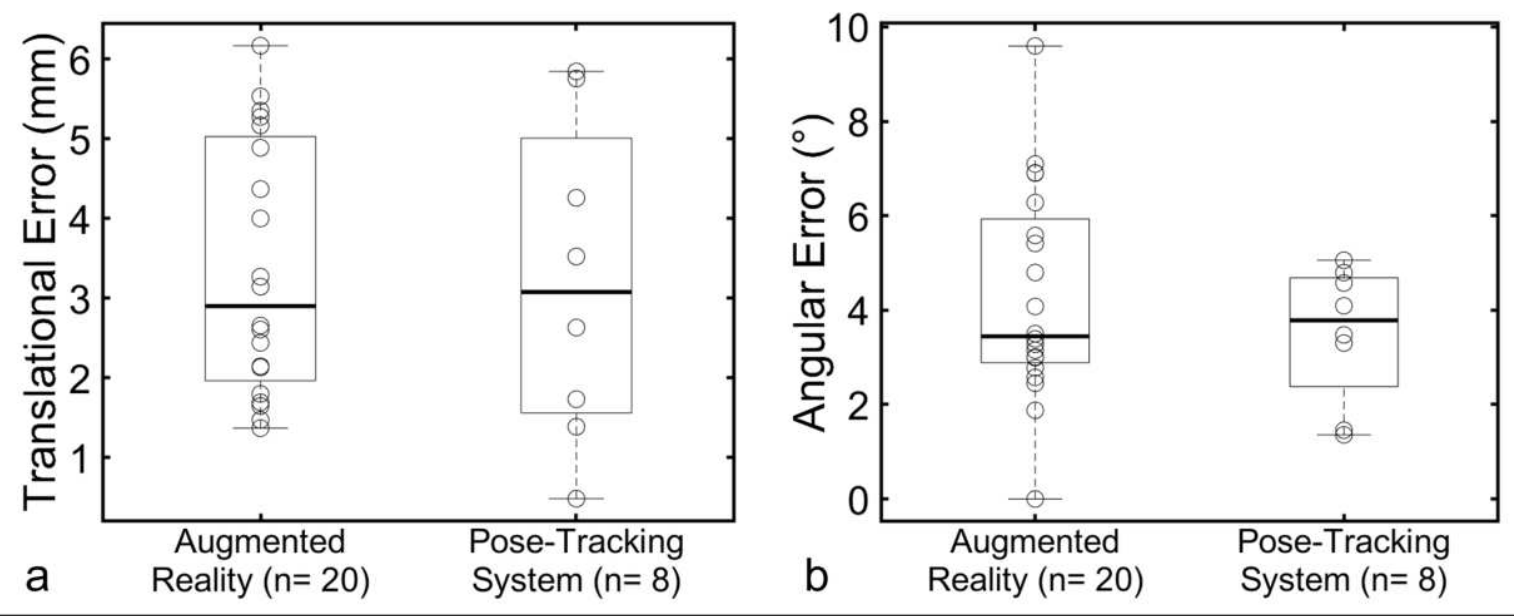

Figure 6 - Results: Box plots of a) translational error and b) angular error for AR (left) and PTS (right) groups.

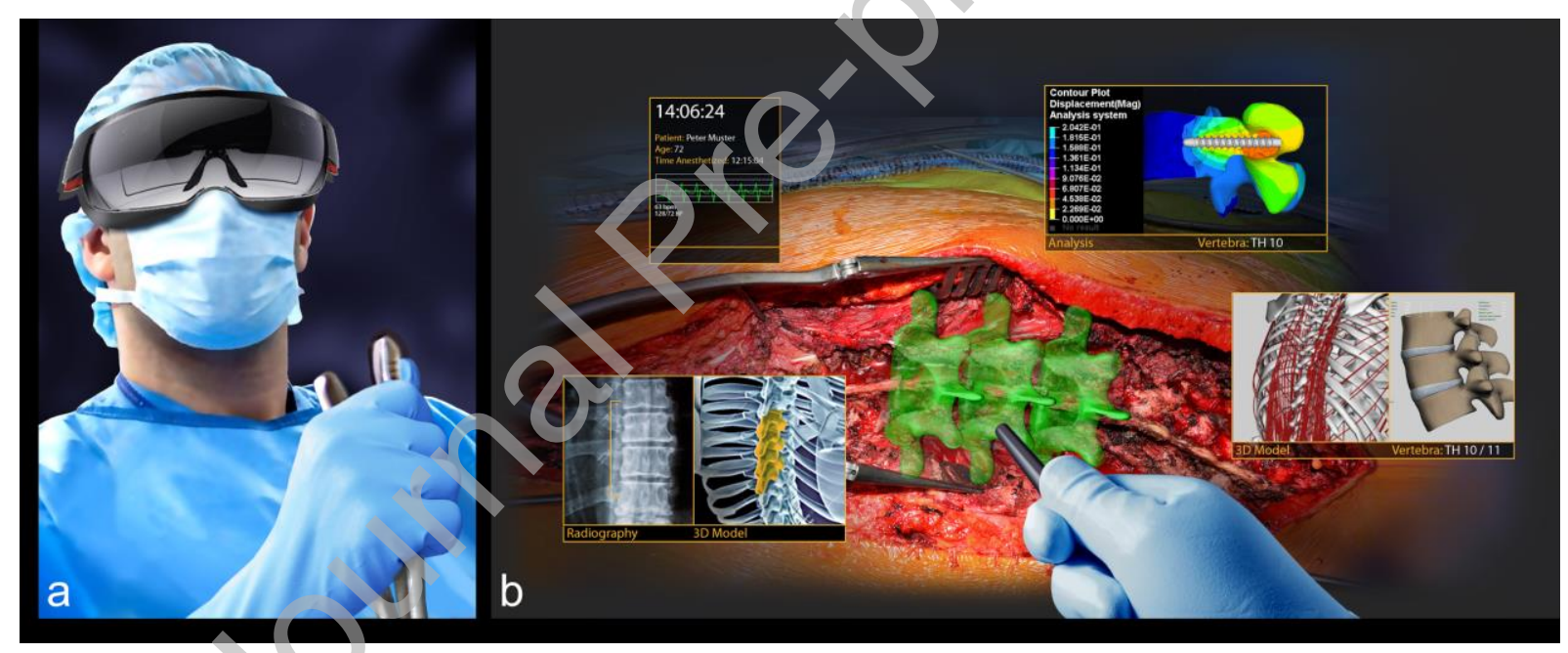

Figure 7 - AR-enhanced surgery: Our vision of AR applied as surgeon enhancing technology. a) Surgeon wearing the HMD as a command control interface. b) Surgeon's augmented view through an HMD. 
Table 1: subjective evaluation of surgical methods

\begin{tabular}{|c|c|c|c|}
\hline & $\begin{array}{c}\text { Free-hand } \\
\text { (incl. Fluoroscopy) }\end{array}$ & $\mathbf{A} \mathbf{R}^{1}$ & PTS $^{2}$ \\
\hline $\begin{array}{l}\text { visual impression of registration } \\
\text { accuracy }\end{array}$ & $N / A^{3}$ & 3 & 5 \\
\hline comfort of usability & 3 & 4 & 4 \\
\hline intuitiveness of system & 2 & 5 & 4 \\
\hline visualization of entry points & $\mathrm{N} / \mathrm{A}^{3}$ & 3 & 4 \\
\hline visualization of angle navigation & $\mathrm{N} / \mathrm{A}^{3}$ & 4 & 3 \\
\hline time expenditure & 5 & & 4 \\
\hline stability of system & 5 & 3 & 4 \\
\hline feasibility for surgery & 5 & $\mathrm{~N} / \mathrm{A}^{3}$ & 3 \\
\hline preserved sterility of surgical site & 3 & 5 & 5 \\
\hline Mean & $\mathbf{N} / \mathbf{A}^{3}$ & 3.4 & 4.0 \\
\hline
\end{tabular}

Scoring from 1-5, whereas 1 represents very bad, 3 average and 5 very good. 